\title{
TABLA DE VIDA Y FECUNDIDAD DE NEZARA VIRIDULA VAR. SMARAGDULA (HEMIPTERA: PENTATOMIDAE) ALIMENTADA SOBRE FRUTOS DE PHASEOLUS VULGARIS L. (FABACEAE) ${ }^{1}$
}

\author{
TABLE OF LIFE AND FECUNDITY BY NEZARA VIRIDULA \\ VAR. SMARAGDULA (HEMIPTERA: PENTATOMIDAE) FEED ON PHASEOLUS \\ VULGARIS L. (FABACEAE) FRUITS
}

\author{
Jorge Omar Werdin González $z^{2}$; Adriana Alicia Ferrero ${ }^{3}$
}

\section{RESUMEN}

El objetivo del presente estudio es determinar la duración del ciclo de vida, proporción sexual, longevidad, fecundidad y tabla de vida horizontal por edades de Nezara viridula var. smaragdula, insecto plaga de la soja alimentado sobre frutos de Phaseolus vulgaris. Para ello se estableció una colonia en laboratorio bajo las siguientes condiciones: temperatura $28 \pm 1{ }^{\circ} \mathrm{C} ; 60 / 70 \% \mathrm{HR}$ y fotoperíodo $14 \mathrm{~L}-10 \mathrm{O}$. Se estudió el ciclo vital y tabla de vida a partir de una cohorte de 4.008 huevos. Los resultados obtenidos fueron: tiempo de desarrollo de los estados inmaduros: huevos-ninfas $49 \pm 4$ día; longevidad de adultos: $41 \pm 9$ días; proporción sexual $\left(\$ / \bigcirc^{7}\right): 1,24$. La tasa de mortalidad específica de cada estadio $\left(\mathrm{q}_{\mathrm{x}}\right)$ fue: huevo: 0,086 ; ninfa I: 0,173; ninfa II: 0,366; ninfa III: 0,540; ninfa IV: 0,426; ninfa V: 0,601; la fecundidad $\left(\mathrm{m}_{\mathrm{x}}\right): 112$ huevos/hembra y el índice reproductor neto $\left(\mathrm{R}_{0}\right): 5,6$. Estos parámetros resultan de interés no sólo para conocer la dinámica poblacional en laboratorio sino para diseñar estrategias de control de esta plaga.

Palabras clave: Tabla de vida, fecundidad, Nezara viridula var. smaragdula.

\begin{abstract}
The purpose of this work is to determine the length of life cycle, sex ratio, longevity, fecundity and horizontal table of life by age of the Nezara viridula var. smaragdula, insect pest of soybean fed on fruits of Phaseolus vulgaris. Thus, the following parameters were carried out the breeding of the insect, its life cycle and life table (cohort 4008 eggs) in laboratory, under the following conditions: temperature $28 \pm 1{ }^{\circ} \mathrm{C}$; relative humidity $60 / 70 \%$ and photoperiods: 14 Light - 10 Dark. The results were as follows: the developmental period of eggs and nymphs were $49 \pm 4$ days; the adult longevity was $41 \pm 9$ days; with a sexual proportion: $\left(0 / \sigma^{7}\right): 1,24$. Specific mortality rate for each stage $\left(q_{x}\right)$ was: egg: 0,086; nymph I: 0,173; nymph II: 0,366; nymph III: 0,540; nymph IV: 0,426; nymph V: 0,601; fecundity $\left(m_{x}\right): 112$ eggs/female and net reproductive rate $\left(R_{0}\right): 5,6$. These parameters are interest to know the population dynamics in laboratory and to design control strategies of this pest.
\end{abstract}

Key words: Table of life, fecundity, Nezara viridula var. smaragdula.

\section{INTRODUCCIÓN}

Nezara viridula (Linnaeus, 1758) es una especie cosmopolita y polimórfica. De las nueve variedades descriptas, tres son las principales: var. smaragdula Fabricius, var. torquata Fabricius y var. aurantiaca Costa (Viván y Panizzi, 2006).
$N$. viridula es una especie polífaga habiéndose identificado hasta el momento más de 100 especies diferentes de plantas pertenecientes a más de 30 familias que actúan como hospederas y entre las cuales figura la soja (Glycine max) como la fuente de alimento preferencial de este insecto (Pannizi, 2000; Kristen y Geoff, 2006).

El artículo forma parte de una tesis doctoral.

2 Universidad Nacional del Sur, Depto. de Biología, Bioquímica y Farmacia. Lab. Zoología de Invertebrados II. San Juan 670 (8000) Bahía Blanca, Argentina. E-mail: jwerdin@ hotmail.com

3 Universidad Nacional del Sur, Depto. de Biología, Bioquímica y Farmacia. Lab. Zoología de Invertebrados II San Juan 670 (8000) Bahía Blanca, Argentina. E-mail: aferrero@uns.edu.a 
Dentro de las chinches que atacan a este cultivo, $N$. viridula es considerada la plaga más importante porque al alimentarse directamente de las semillas reduce la calidad y el potencial germinativo de las mismas, afectando la producción (Panizzi et al., 2000; Corrêa-Ferreira y Azevedo, 2002). Las ninfas I y II tienen hábitos gregarios, no produciendo daños en la planta. A partir de las ninfas III se inicia el periodo normal de alimentación (Corpuz, 1969). En la soja, las ninfas del cuarto y quinto estadio son las responsables de la dispersión de esta plaga. Asimismo se ha señalado que las formas adultas causan más daños en la producción que las ninfas IV y V (McPherson et al., 1979).

La producción de soja (Glycine max) en Argentina se incrementó a partir 1970 hasta alcanzar, según datos de la Secretaría de Agricultura, Ganadería, Pesca y Alimentos en la campaña 2003/2004, una producción total de 31.554.251 ton, siendo el área sembrada de 14.509.306 ha y el rinde por hectárea de $2.209 \mathrm{~kg}$. En la Provincia de Buenos Aires estos valores fueron de $3.205 .523 \mathrm{ha} / \mathrm{siembra}, 3.133 .613$ ha/cosechadas, 7.852.000 toneladas y un rinde de $2.506 \mathrm{~kg} / \mathrm{ha}$. Esto convierte a la Argentina en el tercer productor mundial de este grano, el primer exportador mundial de aceite de soja y el segundo de harina de soja. Dicho cultivo representa en la actualidad el rubro de exportación de mayor incidencia en el Producto Bruto Agropecuario del país, y el mayor generador de divisas (SAGPyA, 2006).

Debido a la importancia de esta plaga, muchos estudios se han llevado a cabo en el mundo con el objeto de mitigar los daños en los cultivos (Seymour $e t$ al., 1995). Además varios de ellos están relacionados con la alimentación de $N$. viridula en el laboratorio con el objeto de obtener individuos para bioensayos (Corrêa-Ferreira y Panizzi, 1999).

En consecuencia, el objetivo de este trabajo fue determinar la duración del ciclo de vida, proporción sexual, longevidad, fecundidad y tabla de vida horizontal por edades de Nezara viridula var. smaragdula alimentada sobre frutos de Phaseolus vulgaris, fuente nutricional menos preferida para su desarrollo.

\section{MATERIALES Y MÉTODOS}

\section{CRÍA EN LABORATORIO}

Se efectuó en una sala de cría acondicionada a $28 \pm 1{ }^{\circ} \mathrm{C}, 60 / 70 \% \mathrm{HR}$ y fotoperiodo $14 \mathrm{~L}-10 \mathrm{O}$.
La colonia se inició con adultos invernantes procedentes de la localidad de Rivera $\left(27^{\circ} 09^{\prime}\right.$ $38^{\prime \prime}$ S, 63 14' 48" O), partido de Adolfo Alsina, Provincia de Buenos Aires, Argentina. Se colocaron en recipientes plásticos ( $13 \mathrm{~cm}$ de diámetro x $20 \mathrm{~cm}$ de altura), se alimentaron con frutos de Phaseolus vulgaris y como fuente de agua se les ofreció un algodón embebido. Como refugio de las chinches y sustrato de oviposición se les proporcionó un papel parafinado. Tanto el alimento como el papel se renovaron cada tres días.

Los huevos se colocaron en cajas de Petri plásticas ( $9 \mathrm{~cm}$ de diámetro) conteniendo en la base papel de filtro y un algodón humedecido. Se tuvo la precaución de que en cada caja no se superaran los 100 huevos, para evitar los efectos de la densidad.

A partir del estadio de ninfa II se agregó a las cajas de Petri frutos de Phaseolus vulgaris. En el estadio de ninfa III los insectos se transfirieron a recipientes plásticos ( $12 \mathrm{~cm}$ de alto x $12 \mathrm{~cm}$ de diámetro), permaneciendo allí hasta alcanzar el estado adulto. Se mantuvieron las mismas condiciones de alimentación para todos los estados mencionados. Cuando los insectos mudaron al estado de adulto se transfirieron en forma definitiva a las jaulas de cría.

Producida la primera generación de adultos en laboratorio se evaluó la proporción sexual.

\section{CICLO DE VIDA}

De 4.008 huevos obtenidos en la primera generación de laboratorio, se siguió su desarrollo, llevándose un registro del tiempo de duración de los distintos estados y estadios ninfales.

\section{CARACTERÍSTICAS REPRODUCTIVAS. CÁLCULO DE LA FECUNDIDAD}

En quince parejas individualizadas se llevó el registro de fecha de nacimiento, duración de los períodos de preoviposición, oviposición y postoviposición, longevidad y número total de huevos colocados por hembra.

\section{TABLA DE VIDA HORIZONTAL POR EDADES}

Las tablas de vida representan, por un lado, una manera sinóptica y sintética de plasmar en forma cuantitativa y numérica las principales características de la mortalidad específica por edades y, por otro, 
es un punto de partida para elaborar parámetros poblacionales que permiten establecer características concernientes a la población en estudio (Rabinovich, 1980; Begon et al., 1988).

Para su realización se partió de una cohorte de 4.008 huevos, estableciéndose para cada intervalo de edad los siguientes parámetros:

$\mathbf{N}_{\mathbf{x}}$ : Número total de individuos observados al inicio de cada estado o estadío. $1_{\mathrm{x}}$ : Proporción de la cohorte original que sobrevive al inicio de cada estado o estadio: $\left(\mathrm{N}_{\mathrm{x}} / \mathrm{N}_{0}\right)$.

$\mathbf{d}_{\mathbf{x}}$ : Proporción de la cohorte original que muere en cada estadio $d_{x}=1_{x}-1_{x+1} q_{x}$ : Tasa de mortalidad $\left(\mathrm{d}_{\mathrm{x}} / \mathrm{l}_{\mathrm{x}}\right)$

K: Fuerza de mortalidad $k=\log _{10} \mathrm{~N}_{\mathrm{x}}-\log _{10} \mathrm{~N}_{\mathrm{x}+1}$

$\mathbf{m}_{\mathbf{x}}$ : Número de huevos producidos por cada hembra sobreviviente.

$\mathbf{F}_{\mathbf{x}}$ : Número total de huevos producidos en el estado adulto.

$\mathbf{R}_{\mathbf{o}}$ : Índice reproductor neto $\mathrm{R}_{0}=1_{\mathrm{x}} \mathrm{m}_{\mathrm{x}}$

\section{RESULTADOS Y DISCUSIÓN}

En la primera generación de adultos de $N$. viridula var. smaragdula alimentada sobre Phaseolus vulgaris en laboratorio se obtuvieron 201 individuos adultos, de los cuales 112 resultaron hembras y 90 machos, siendo la relación de sexos $($ Q/O'): 1,24 .

En la Tabla 1 se presenta la duración de los distintos estados y estadios de Nezara viridula var. smaragdula.

La duración promedio en días de los períodos de preoviposición, oviposición y posoviposición, longevidad de adultos ( $\left(\right.$ $\left.-\bigcirc^{7}\right)$ y número de huevos colocados por hembra, a partir de 15 parejas Nezara viridula var. smaragdula se registra en la Tabla 2.
Tabla 1

Duración en días de los estadios y estados biológicos de Nezara viridula var. smaragdula a temperatura $28 \pm 1{ }^{\circ} \mathrm{C}$, humedad relativa $60 / 70 \%$, fotoperíodo $14 \mathrm{~L}-10 \mathrm{O}$ $(n=4.008)$

\begin{tabular}{|l|c|}
\hline \multicolumn{1}{|c|}{ Estado/Estadio } & Total días \\
\hline Huevo & $6,388 \pm 0,687$ \\
\hline Ninfa I & $5,638 \pm 1,046$ \\
\hline Ninfa II & $7,111 \pm 1,282$ \\
\hline Ninfa III & $8,583 \pm 1,574$ \\
\hline Ninfa IV & $8,277 \pm 2,814$ \\
\hline Ninfa V & $13,116 \pm 3,093$ \\
\hline Adulto & $41,433 \pm 9,257$ \\
\hline Total estados inmaduros & $49,117 \pm 3,856$ \\
\hline Total Huevo - Adulto & $90,55 \pm 3,625$ \\
\hline
\end{tabular}

En la Tabla 3 se muestran los valores de los parámetros poblacionales registrados para Nezara viridula var. smaragdula.

En base a los datos obtenidos sobre la duración en días de los estadios y estados biológicos de Nezara viridula var. smaragdula se puede inferir que en las condiciones establecidas el ciclo se cumple en $90 \pm 4$ días. La longevidad promedio de los adultos es casi la mitad de la vida total. No se observaron diferencias en la duración de los estadios de ninfa I a ninfa IV. El estadio de ninfa $\mathrm{V}$ mostró una duración de 13 días, tiempo superior a los estadios anteriores (Tabla 1). Harris y Todd (1980a) al estudiar la duración de los estadios de Nezara viridula a una temperatura entre $25^{\circ} \mathrm{C}$ y $28^{\circ} \mathrm{C}$, y un fotoperíodo de $14 \mathrm{~L}: 100$ con dieta basada en granos de Phaseolus vulgaris y maní obtuvieron resultados similares a nuestro estudio sólo en lo referido a la duración de estado de huevo, ninfa IV y V. Noda y Kamano

Tabla 2

Duración media en días de los periodos de pre, pos y oviposición, vida adulta y número de huevos por hembra de Nezara viridula var. smaragdula a temperatura $28 \pm 1^{\circ} \mathrm{C}$, humedad relativa $60 / 70 \%$, fotoperíodo $14 \mathrm{~L}-10 \mathrm{O}$.

\begin{tabular}{|c|c|c|c|c|c|}
\hline \multirow{2}{*}{ Preoviposición } & \multirow{2}{*}{ Oviposición } & \multirow{2}{*}{ Posoviposición } & \multirow{2}{*}{$\begin{array}{c}\text { No total de huevos } \\
\text { ( }\end{array}$} & & \multicolumn{2}{|c|}{ Duración de vida } \\
\cline { 4 - 6 } & & & 9 & $\sigma^{\top}$ \\
\hline $24 \pm 9 \mathrm{a}$ & $7 \pm 11 \mathrm{~b}$ & $16 \pm 7 \mathrm{c}$ & $112 \pm 44$ & $47 \pm 8 \boldsymbol{a}$ & $36 \pm 5 \boldsymbol{b}$ \\
\hline
\end{tabular}

Los valores son las medias sin transformar \pm ES. Valores seguidos por la misma letra no difieren significativamente (DMS, $\mathrm{p}>0,05)$. 
Tabla 3

Tabla de vida horizontal de una cohorte $(n=4008)$ de Nezara viridula var. smaragdula a temperatura $28 \pm 1{ }^{\circ} \mathrm{C}$, Humedad Relativa 60/70\%, fotoperíodo $14 \mathrm{~L}-10 \mathrm{O}$

\begin{tabular}{|l|c|c|c|c|c|c|c|c|}
\hline \multicolumn{1}{|c|}{ Estado - Estadio } & $\mathbf{N}_{\mathbf{x}}$ & $\mathbf{I}_{\mathbf{x}}$ & $\mathbf{d}_{\mathbf{x}}$ & $\mathbf{q}_{\mathbf{x}}$ & $\mathbf{K}$ & $\mathbf{F}_{\mathbf{x}}$ & $\mathbf{m}_{\mathbf{x}}$ & $\mathbf{R}_{\mathbf{o}}$ \\
\hline Huevo & 4.008 & 1,000 & 0,086 & 0,086 & 0,040 & & & \\
\hline Ninfa I & 3.660 & 0,913 & 0,158 & 0,173 & 0,083 & & & \\
\hline Ninfa II & 3.024 & 0,754 & 0,276 & 0,366 & 0,198 & & & \\
\hline Ninfa III & 1.917 & 0,478 & 0,258 & 0,540 & 0,337 & & & \\
\hline Ninfa IV & 882 & 0,220 & 0,0938 & 0,426 & 0,241 & & & \\
\hline Ninfa V & 506 & 0,126 & 0,076 & 0,601 & 0,399 & & & \\
\hline Adulto & 202 & 0,050 & - & - & - & 12.544 & 112 & 5,6 \\
\hline
\end{tabular}

(2002) evaluando el tiempo de desarrollo de huevo a adulto de $N$. viridula a una temperatura de $25^{\circ} \mathrm{C}$, fotoperiodo de $16 \mathrm{~L}: 80$ y alimentadas sobre maní y soja registraron una duración de 26 días inferior a lo observado en nuestro estudio. Es sabido que el tiempo de desarrollo ninfal en $N$. viridula disminuye con el incremento de la temperatura y la longitud del fotoperiodo (Vivan y Panizzi, 2005).

Del análisis del comportamiento reproductivo se observaron diferencias significativas entre los periodos de preoviposición, oviposición y posoviposición en $N$. viridula var. smaragdula $(\mathrm{p}<0,05)$ (Tabla 2). Panizzi y Mourao (1999) al alimentar individuos adultos de $N$. viridula con frutos de soja en condiciones similares de cría, registraron un tiempo de preoviposición semejante a nuestro estudio, sin embargo cuando utilizaron frutos de Ligustrum lucidum el período fue de 15,5 días.

La duración del período de oviposición fue relativamente corto en comparación con la duración total de la vida de la hembra (15\%). Además, el tiempo de vida de los machos fue inferior al de las hembras ( $\mathrm{p}<0,05)$ (Tabla 2). Harris y Todd (1980b) contabilizaron un número de huevos superior por hembra cuando los adultos eran alimentados con granos de Phaseolus vulgaris y maní. El número de huevos puestos por hembra de $N$. viridula var. smaragdula alimentados con frutos de Phaseolus vulgaris en nuestro trabajo, no difiere de lo observado por Panizzi y Mourao (1999) al alimentar los insectos con soja. Sin embargo, Viván y Panizzi (2005) alimentado con brotes de soja a N. viridula var. smaragdula en las mismas condiciones de temperatura, humedad y fotoperíodo que en nuestro estudio obtuvieron un menor número de huevos / hembra.

Los parámetros poblacionales registrados en la tabla de cohorte (Tabla 3) muestran que la tasa de mortalidad $\left(\mathrm{q}_{\mathrm{x}}\right)$ es baja en el estado de huevo y el estadio de ninfa I. Dado que las ninfas I no se alimentan, esto estaría indicando que las condiciones de temperatura, humedad y fotoperíodo utilizadas en nuestro ensayo serían las adecuadas para el desarrollo de estos estados.

La intensidad de mortalidad (K), como así también $\mathrm{q}_{\mathrm{x}}$, comienzan a aumentar a partir del estadio de ninfa II presentando valores altos en el estadio de ninfa III y ninfa V (Tabla 3). Panizzi (2000) indica que cuando $N$. viridula se alimenta con fuentes nutricionales de menor preferencia se produce una mortalidad del $80 \%$ al $100 \%$ en el estado ninfal, en tanto que sobre Glycine max estos valores oscilan en el 30\%. En consecuencia, la mortalidad observada en los estados ninfales de $N$. viridula var. smargadula podría deberse a que Phaseolus vulgaris es una planta de menor preferencia para esta variedad.

$N$. viridula var. smaragdula alimentada sobre Phaseolus vulgaris mostró una alta fecundidad $\left(\mathrm{m}_{\mathrm{x}}=112\right)$ y un índice reproductor neto elevado $\left(R_{0}=5.6\right)$. Sin embargo, $q_{x}, K$ y la duración del ciclo de vida se vieron afectados por este tipo de alimento. Probablemente un cambio en la dieta con incorporación de otros nutrientes mejoraría estos parámetros, ya que a pesar de que $N$. viridula es una especie polífaga, cuando utiliza como fuente de alimento plantas no preferenciales, los mismos se ven afectados. 


\section{LITERATURA CITADA}

BEGON, M.; HARPER J. L.; COLIN, C. R. 1988. "Ecología: individuos, poblaciones y comunidades. Ed. Omega S.A. 1: 883 .

CORPUZ, L.R. 1969. "The biology, host range and natural enemies of Nezara viridula L. (Hemiptera: Pentatomidae)". Philippine Entomologist. 1(3): 225.

CORRÊA-FERREIRA, B.S.; AZEVEDO, J. 2002. "Soybean seed damage by different species of stink bugs". Agriculture and Forest Entomology: 4: 145.

CORRÊA-FERREIRA, B. S.; PANIZZI,A. R. 1999. "Percevejos da soja e seu manejo". Londrina: Embrapa-CNPSo. Circular Técnica 24: 45.

HARRIS, V.E.; TODD, J.W. 1980a. "Comparative fecundity, egg fertility and haych among wild-type and three laboratory reared generations of the southern green stink bug, Nezara viridula (L.) (Hemiptera: Pentatomidae)". J. Georgia Entomol. Soc. 15 (3): 241-245.

HARRIS, V.E.; TODD, J.W. 1980b. "Duration of the inmature stages of the green stink bug, Nezara viridula (L.) with a comparative reviews of previous studies)". J. Georgia Entomol. Soc. 15 (2) 109-114.

KRISTEN M. M. K., GEOFF M. G. 2006. "Review of Nezara viridula (L.) management strategies and potential for IPM in field crops with emphasis on Australia". Crop. Protection. Artículo en prensa.

McPHERSON, R.M.; NEWSON, L.D.; FARTHING, B.F. 1979. "Evaluation of four stink bug species from three general affecting soybean yield and quality in Lousiana". Journal of Economic Entomology. 72: 188.

NODA, T.; KAMANO, S. 2002. "Artificial rearing of Nezara viridula (L.) and N. antennata Scott (Heteroptera:
Pentatomidae) with semi-solid meridic diets". Appl. Entomol. Zool. 37 (1): 43-50.

PANIZZI A. R. 2000. "Suboptimal Nutrition and Feeding Behavior of Hemipterans on Less Preferred Plant Food Sources". An. Soc. Entomol. Brasil 29(1): 1-12.

PANIZZI, A. R.; MCPHERSON, J.E.; JAMES, D.G.; JAVAHERY, M.; MCPHERSON, R.M. 2000. "Stink bugs (Pentatomidae)". In: Schaefer, C.W.; panizzi, A.R. (eds.). Heteroptera of economic importance. Boca Raton, Florida, USA; CRC Press. 432-434.

PANIZZI, A. R.; MOURÃO, A. P. M. 1999. "Mating, ovipositional rhythm and fecundity of Nezara viridula (L.) (Heteroptera: Pentatomidae) fed on privet, Ligustrum lucidum Thunb. and on soybean, Glycine $\max$ (L.) merrill fruits". An. Soc. Entomol. Brasil 28(1): 35-40.

RABINOVICH J. 1980. "Introducción a la Ecología de Poblaciones Animales”. Ed. Continental S.A. México. 1-313.

SAGPYA: SECRETARIA DEAGRICULTURA, GANADERIA, PESCA Y ALIMENTOS 2006. www.sagpya.mecon. gov.ar

SEYMOUR, J.; BOWMAN, G.; CROUCH, M. 1995. Effects of neem tree extract on feeding frequency of Nezara viridula L. (Hemiptera: Pentatomidae) on pecan nuts. Australian Entomological Society Journal, 34 (1): 221-223.

VIVAN L. M.; PANIZZI A. R. 2006. "Geographical distribution of genetically determined types of Nezara viridula (L.) (Heteroptera: Pentatomidae) in Brazil". Neotrop. Entomol. 35 (2): 175-181. 
